\title{
MULTILEVEL FILTERING PRECONDITIONERS: EXTENSIONS TO MORE GENERAL ELLIPTIC PROBLEMS*
}

\author{
CHARLES H. TONG ${ }^{\dagger}$, TONY F. CHAN ${ }^{\ddagger}$, AND C. C. JAY KUO $\$$
}

\begin{abstract}
The concept of multilevel filtering (MF) preconditioning applied to second-order selfadjoint elliptic problems is briefly reviewed. It is then shown how to effectively apply this concept to other elliptic problems such as the second-order anisotropic problem, biharmonic equation, equations on locally refined grids and interface operators arising from domain decomposition methods. Numerical results are given to show the effectiveness of the MF preconditioners on these problems.
\end{abstract}

Key words. multilevel preconditioners, elliptic problems, conjugate gradient method, domain decomposition

AMS(MOS) subject classifications. $65 \mathrm{~F} 10,65 \mathrm{~N} 30$

1. Introduction. Preconditioned conjugate gradient (PCG) methods have been a very popular and successful class of methods for solving large systems of equations arising from discretizations of elliptic partial differential equations. With the advent of parallel computers in recent years, there has been increased research into effective implementation of these methods on various parallel computers. Since effective preconditioning plays a critical role in the competitiveness of the PCG methods, many classical preconditioners have been proposed and studied, especially for second-order elliptic problems. Among these are the Jacobi preconditioner (diagonal scaling), the SSOR preconditioner, the incomplete factorization preconditioners (ILU and MILU), and polynomial preconditioners. Many such preconditioners have been very successful in giving high performance, especially when implemented on sequential computers.

In the parallel implementation of PCG methods, the major bottleneck is often the parallelization of the preconditioner. The rest of the PCG methods can usually be parallelized in a straightforward way (the inner product computation is also considered a bottleneck but its wide applicability in other methods has prompted many parallel computer manufacturers to develop a highly optimized and efficient code for it). Unfortunately, for many of the classical preconditioners, there is a fundamental trade-off in the ease of parallelization and the rate of convergence. A principal obstacle to parallelization of many preconditioners that are effective in improving the convergence rate (e.g., SSOR, ILU, and MILU) is the sequential manner these preconditioners use in traversing the computational grid - the data dependence implicitly prescribed by the method limits the amount of parallelism available. Reordering the grid traversal (e.g., from natural to red-black ordering) or inventing new methods

* Received by the editors October 4, 1990; accepted for publication (in revised form) October 25, 1990. This work was supported in part by the Department of Energy contract DE-FG-03-87-ER25037, the Army Research Office contract DAAL03-88-K-0085, and the National Science Foundation grant contract FDP NSF ASC 9003002.

† Sandia National Laboratories, Livermore, California 94551-0969 (chtong@snll-arpagw.llnl.gov). The research of this author was supported by a Defense Advanced Research Projects Agencysponsored graduate research assistantship (award 26947F) through the University of Maryland Institute of Advanced Computer Studies.

$\ddagger$ Department of Mathematics, University of California, Los Angeles, California 90024 (chan@math.ucla.edu).

$\S$ Department of Electrical Engineering-Systems, University of Southern California, Los Angeles, California 90089 (cckuo@sipi.usc.edu). The research of this author was supported by the University of Southern California Faculty Research and Innovation Fund and a National Science Foundation Research Initiation Award. 
(e.g., polynomial preconditioners) to improve the parallelization alone often has an adverse effect on the rate of convergence [8].

The fundamental difficulty can be traced to the global dependence of elliptic problems. An effective preconditioner must account for the global coupling inherent in the original elliptic problem. Preconditioners that use purely local information (such as red-black orderings and polynomial preconditioners) are limited in their ability to improve the convergence rate. On the other hand, global coupling through a naturally ordered grid traversal is not highly parallelizable. The challenge is therefore to construct effective global coupling that is highly parallelizable. We are thus led to the consideration of preconditioners that share global information through a multilevel grid structure (ensuring a good convergence rate) but perform only local operations on each grid level (and are hence highly parallelizable). Preconditioners of the multilevel type for second-order selfadjoint operators have been proposed recently by several researchers, including Bramble, Pasciak, and Xu [6]; Axelsson [1]; Vassilevski [25]; Axelsson and Vassilevski [2]; [3]; Kuo, Chan, and Tong [13]; and Kuznetsov [14].

The main goal of our paper is to employ the main ideas in [6] and [13] to develop algorithms for more general problems, such as second-order anisotropic problems, the biharmonic equation, problems on locally refined grids, and interface operators for domain decomposition methods. Our approach can be viewed as adapting the projection operators and eigenvalue estimates in [6] to more general problems. On the other hand, the filtering framework of [13] offers the flexibility in designing the filters (or projection operators), which improves the performance substantially in several cases (e.g., the anisotropic case). In particular, the second-order anisotropic problems and problems on locally refined grids can be solved more efficiently by using different types of filters, while the the biharmonic equation and interface operator can be solved efficiently by using different eigenvalue estimates. To the best of our knowledge, two of these extensions (i.e., the biharmonic and the domain decomposition applications) are novel. While a general theory is lacking at this point, we demonstrate numerically that these algorithms perform very well, at least for model problems.

The multilevel preconditioners mentioned above are similar in spirit to the classical multigrid method. They are designed to capture the mesh-dependent spectral property of a discretized elliptic operator. The variations in the coefficients are handled in most cases by the conjugate gradient method, which also makes the iteration more robust. In [13], we presented some experimental results comparing several multilevel preconditioners with a multigrid cycle as a preconditioner. However, further tests are needed to decide whether this new class of multilevel preconditioners offers practical advantages over the classical multigrid methods.

2. The concept of MF preconditioners. We shall motivate the construction of the MF preconditioner by first considering the following one-dimensional Poisson equation on $\Omega=[0,1]$ :

$$
-\triangle u=f(x)
$$

subject to zero Dirichlet boundary conditions. A standard second-order discretization of the above equation on a uniform grid with grid size $h=1 /(n+1)$ gives rise to a linear system of equations denoted by $A u=f$, where $A, u$, and $f$ correspond to the discrete Laplacian, the solution and the forcing functions, respectively, and $A$ is a tridiagonal matrix with diagonal elements $-1 / h^{2}, 2 / h^{2},-1 / h^{2}$. It is well known that the matrix $A$ can be diagonalized as

$$
A=W \Lambda W^{T}
$$


where $W$ is an orthogonal matrix with elements

$$
(W)_{i j}=2 \sqrt{h} \sin i j \pi h
$$

and

$$
\Lambda=\operatorname{diag}\left(\lambda_{k}\right), \quad \lambda_{k}=\frac{4}{h^{2}} \sin ^{2} \frac{k \pi h}{2} .
$$

The main idea of the MF preconditioning is to approximate this eigendecomposition of $A$. First, the eigenfunctions of $A$ are grouped into subsets corresponding to different frequency bands. In matrix form, for $n=2^{L}-1$, we partition $W$ into $L$ bands so that

$$
W=\left[W_{1}, W_{2}, \cdots, W_{L}\right]
$$

where

$$
W_{l}=\left[w_{2^{l-1}}, \cdots, w_{2^{l}-1}\right]
$$

with $w_{j}$ being the $j$ th column of the matrix $W$. Thus, for example, $W_{1}$ and $W_{L}$ correspond to the lowest and highest frequency bands, respectively.

Using the notations introduced above, we can rewrite

$$
A=\sum_{l=1}^{L} W_{l} \Lambda_{l} W_{l}^{T}
$$

where

$$
\Lambda=\operatorname{diag}\left(\Lambda_{l}\right), \Lambda_{i}=\operatorname{diag}\left(\lambda_{2^{l-1}}, \cdots, \lambda_{2^{l}-1}\right) .
$$

The first approximation comes in when we replace all the eigenvalues $\left(\Lambda_{l}\right)$ within each band by a constant $c_{l}$. Thus, we have a preconditioner $\hat{M}$ such that

$$
\hat{M}^{-1} v=\sum_{l=1}^{L} \frac{B_{l} v}{c_{l}}
$$

where

$$
B_{l}=W_{l} W_{l}^{T}
$$

Note that we have the following property for $B_{l}$ :

$$
B_{l} v= \begin{cases}v & \text { if } v \in \text { range }\left\{W_{l}\right\} \\ 0 & \text { if } v \in \text { range }\left\{W_{l}\right\}^{\perp} .\end{cases}
$$

Hence, $B_{l}$ can be considered as an ideal spatial bandpass filter. Thus, applying the preconditioner $\hat{M}$ to a vector (i.e., $M^{-1} v$ ) consists of three phases : projection of $v$ into the subspace corresponding to each band (operator $B_{l}$ ), scaling by the corresponding approximate eigenvalues $c_{l}$, and synthesizing the scaled components (summation).

Since the implementation of ideal filters is computationally expensive, requiring many global operations (e.g., sine transforms), we seek the approximation of ideal filters with nonideal ones that are computationally more efficient. For the construction 
of efficient nonideal filters, we borrow ideas from standard digital filtering theory [13]. Typically, a bandpass filter is constructed by taking the difference of two lowpass filters, one that filters out all frequencies higher than the highest ones in the band and the other one that lets through all frequencies lower than all frequencies in the band. In turn, the lowpass filters can be approximated by cascading a sequence of elementary filters $H_{l}$ 's, which are simple averaging operators over a small fixed number of grid points separated by spacing proportional to the wavelength of the band $W_{l}$.

Mathematically, the effect of using nonideal filters can be summarized by replacing $B_{l}$ with approximations $\tilde{B}_{l}$ in the definition of $\hat{M}$ to get our final preconditioner $M$ :

$$
M^{-1} v=\sum_{l=1}^{L} \frac{\tilde{B}_{l} v}{c_{l}} .
$$

In the rest of the paper, we use the following two filters :

- The first order filter defined by

$$
\left(H_{l, 1}\right)_{j}=\frac{1}{4}\left(v_{j-2^{L-l}}+2 v_{j}+v_{j+2^{L-l}}\right)
$$

where $(\cdot)_{j}$ denotes the $j$ th element of the argument, and $v$ is extended periodically by

$$
v_{-j}=-v_{j}, \text { and } \quad v_{n+j}=-v_{n+2-j} .
$$

- The filter $H_{l, 2}$ obtained by applying $H_{l, 1}$ twice :

$$
\left(H_{l, 2}\right)_{j}=\frac{1}{16}\left(v_{j-2^{L-l+1}}+4 v_{j-2^{L-l}}+6 v_{j}+4 v_{j+2^{L-l}}+v_{j+2^{L-l+1}}\right) .
$$

We call the method introduced above the single grid multilevel filtering (SGMF) preconditioner, which involves computation on the same number of grid points $n$ at all levels (corresponding to the frequency bands). Since there are $L=\log _{2}(n+1)$ levels and $O(n)$ operations are required per level, the total number of operations required per iteration is thus $O(n L)$.

To further improve the efficiency, we introduce a multigrid version of our preconditioner, which we called the multigrid multilevel filtering (MGMF) preconditioner. This is motivated by the fact that waveforms consisting only of low wavenumber components can be well represented on coarser grids. To incorporate the multigrid structure, the operators $I_{l+1}^{l}$ and $I_{l-1}^{l}$, which are the down-sampling and up-sampling operators, respectively, are introduced. Note that in the multigrid literatures these operators are commonly known as restriction and interpolation operators. Using the concept of MGMF, we construct a sequence of grids $\Omega_{l}$ of sizes $h_{l}=O\left(2^{L-l} h\right), 1 \leq l \leq L$, to represent the decomposed components. With MGMF, the total number of operations per iteration is $O(n)$, a reduction by a factor of $\log _{2} n$ compared to SGMF.

We allow variations in designing the filtering scheme. Several preconditioners, which will be used in the later sections, are defined specifically as follows:

MGMF1, the MGMF preconditioner with 9-point (27-point) filter for two-dimensional (three-dimensional) problems (i.e., $H_{l, 1}$ ).

MGMF2, a modified version of MGMF in which the 9-point (27-point) filter is applied twice (i.e., $H_{l, 2}$ ). 
MGMF3, another modified version of MGMF in which the 9-point (27-point) filter is applied once at the finest grid level (to give a smaller amount of work compared to MGMF2) and twice at other grid levels (to achieve a convergence rate between MGMF1 and MGMF2 but close to MGMF2).

We summarize the MGMF1 preconditioning algorithm as follows:

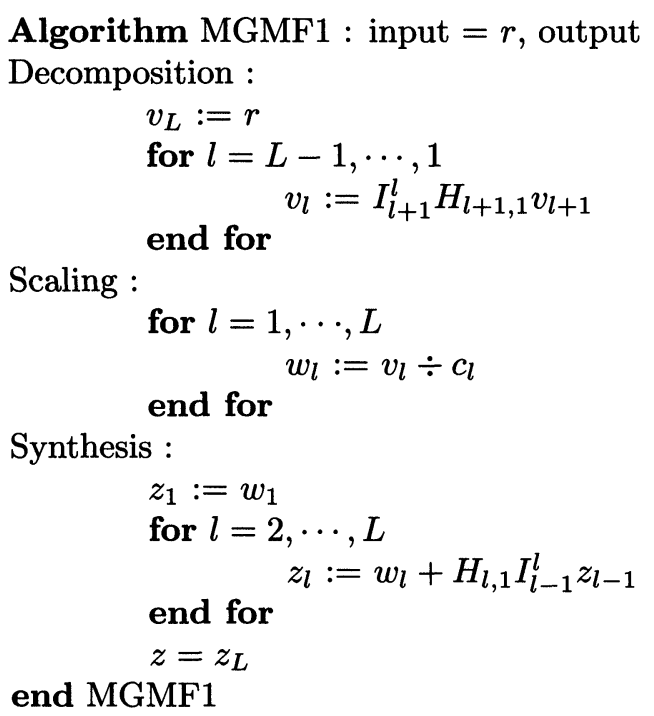

As it stands, this definition of the preconditioner can be extended to higher dimensions, more general elliptic operators and finite element meshes, as long as we have appropriate definitions for the elementary filters $H_{l}$ 's, the restriction and interpolation operators $I_{l+1}^{l}$ and $I_{l}^{l+1}$, and the $c_{l}$ 's. For example, filters for the high dimensional cases can be constructed from the tensor product of one-dimensional filters. Moreover, it is well known that the eigenvalues $\lambda_{k}$ in the wavenumber band $B_{l}$ behave like $O\left(h_{l}^{-2}\right)$ for general second-order elliptic problems, where $h_{l}$ denotes the grid spacing for level $l[21]$. Therefore, a general rule for selecting the scaling constant $c_{l}$ at grid level $l$ is $c_{l}=O\left(h_{l}^{-2}\right)$. For quasiuniform meshes with a refinement factor of 2 (so that $h_{l} \approx 2 h_{l+1}$ ), this leads to the recurrence relation $c_{l+1}=4 c_{l}$.

By appealing to the framework in [6], it is also possible to construct filters for quasi-uniform structured finite element meshes. This relationship was briefly discussed in [13]. Basically, the projection operator from the fine grid onto a coarser grid used in [6] can be interpreted as a low-pass filter on the fine grid. Therefore, on the one hand the elementary filters $H_{l}$ 's can be derived from the basis functions on the grid hierarchy. On the other hand, the projection operators in [6] can be adapted to special features of a particular problem, with the insight provided by the filtering framework (e.g., for anisotropic problems).

The MF preconditioner is designed to capture the mesh-dependent spectral property of a discretized elliptic operator, but not the variation of its coefficients. In order to take badly scaled variable coefficients into account, we use diagonal scaling [10]. Suppose that the coefficient matrix can be written as

$$
A=D^{1 / 2} \tilde{A} D^{1 / 2}
$$

where we choose $D$ to be a diagonal matrix with positive elements in such a way that 
the diagonal elements of $\tilde{A}$ are of the same order. Then in order to solve $A u=f$, we can solve an equivalent problem $\tilde{A} \tilde{u}=\tilde{f}$, where $\tilde{u}=D^{1 / 2} u$ and $\tilde{f}=D^{-1 / 2} f$, with the MF preconditioner.

The SGMF preconditioner on uniform meshes can be easily analyzed exactly using Fourier analysis, and the predictions agree quite well with experimental results [13], [23]. However, the Fourier analysis is only meant to be used as a tool for deriving and gaining insights into the algorithms and cannnot be extended as a basis for a general convergence theory. While the Fourier analysis is rigorously applicable only for model problems, the derived algorithms are applicable in a more general setting. Fourier analysis does provide precise convergence rate estimates and eigenvalue distributions, which supplements the more general theory. For this reason, it has been used by many authors in studying iterative methods [27], [11], [26]. The MGMF preconditioner on uniform and quasi-uniform grids can be analyzed using the same finite element analysis framework used in [6], although we will not pursue that in this paper.

On a uniform mesh there is an obvious connection between our multilevel filtering idea and wavelets [20], [12]. Wavelets are orthonormal basis functions for squareintegrable functions and are defined on a multilevel structure. These basis functions have compact support in space and almost compact support in the Fourier domain. Thus, wavelets can be considered as efficient bandpass filters. We are currently exploring the use of wavelets in our multilevel filtering preconditioner framework.

3. MF preconditioners for anisotropic problems. In this section, we extend the concept of multilevel filtering to the second-order anisotropic problems. To achieve a high degree of efficiency, the preconditioning step requires some modifications in the design of filters (or the use of a different multilevel nodal basis). We first provide justification for such modifications and then show the condition number as computed by Fourier analysis. Numerical experiments are also included.

Consider the following two-dimensional second-order anisotropic problem:

$$
-\alpha u_{x x}-u_{y y}=f(x, y) \text { in } \Omega=[0,1]^{2},
$$

where $\alpha>1$, with zero Dirichlet boundary conditions. The discretization of the equation using a uniform square mesh with $h=1 /(n+1)$ gives a block-tridiagonal matrix $A$ with an equation of the form $A u=f$. In the Fourier domain, we can express this as

$$
\hat{A}(j, k) \hat{u}_{j, k}=\hat{f}_{j, k}, \quad j, k=1,2, \cdots, n-1
$$

where

$$
\hat{u}_{j, k}=\frac{\sqrt{2}}{n} \sum_{l=1}^{n} \sum_{m=1}^{n} u_{l, m} \sin (j \pi l h) \sin (k \pi m h)
$$

and

$$
\hat{f}_{j, k}=\frac{\sqrt{2}}{n} \sum_{l=1}^{n} \sum_{m=1}^{n} f_{l, m} \sin (j \pi l h) \sin (k \pi m h)
$$

such that

$$
\hat{A}(j, k)=(2+2 \alpha)-2(\alpha \cos j \pi h+\cos k \pi h) .
$$


We can observe from the eigenvalue spectrum of $\hat{A}$ that for $\alpha \gg 1$ the variation in magnitudes of the eigenvalues in the $k$-direction is relatively small compared to that in the $j$-direction. To maintain uniform variation of eigenvalues within each band, we divide more wavenumber bands in the $j$-direction than in the $k$-direction. We call this technique directionally adaptive filtering. This can be done in practice by first performing one-dimensional filtering in the $j$-direction for a number of levels (say, the number of levels $=\gamma$ ), then resuming two dimensional filtering. This is in contrast to performing two-dimensional filtering for all the levels for the nearly isotropic problems described in the last section. Here $\gamma$ depends on $\alpha$ as well as the problem to be solved. For second-order elliptic problems with quasi-uniform grid and $h_{l} \approx 2 h_{l+1}$, it is sufficient to use $\gamma=\operatorname{round}\left(\log _{4} \alpha\right)$. Suppose $\alpha=4$. Then $\gamma=1$ and the modified $H_{l, 1}$ for the finest grid level takes the following stencil form :

$$
\frac{1}{4}\left|\begin{array}{lll}
1 & 2 & 1
\end{array}\right|
$$

while the filters for the other coarse grid levels have a two-dimensional stencil (tensor product of one-dimensional filter, i.e., $H_{l, 1} \times H_{l, 1}$ ).

Note that if the finest level is defined on a $(n+2) \times(n+2)$ grid, then for $\gamma \geq 1$ the next coarse level is defined on a $((n+1) / 2+1) \times(n+2)$ grid instead of a $((n+1) / 2+1) \times((n+1) / 2+1)$ grid for $\gamma=0$. It should also be noted that this modified filtering scheme is analogous to the idea of semi coarsening in the multigrid literature.

We performed Fourier analysis of the single grid version of this scheme (called SGMF1a) on the two-dimensional anisotropic problem with different $\alpha$ and $h$. The condition numbers of the preconditioned system are given in Table 1. For comparison purposes, the condition numbers of the preconditioned system using the unmodified SGMF1 preconditioner are also included. Table 1 shows that this modified scheme is quite effective. For example, for $\alpha=1000$ the condition number grows slowly with $n$, while this is not true for the unmodified SGMF1 preconditioner.

TABLE 1

Condition number for different $\alpha$ and $n$.

\begin{tabular}{|r||r|r|r||r|r|r||r|r|r|}
\hline \multicolumn{1}{|r||}{} & \multicolumn{3}{|c||}{$\alpha=10$} & \multicolumn{3}{c||}{$\alpha=100$} & \multicolumn{3}{|c|}{$\alpha=1000$} \\
\hline$n$ & A & SGMF1a & SGMF1 & A & SGMF1a & SGMF1 & A & SGMF1a & SGMF1 \\
\hline \hline 7 & 25 & 3.8 & 13 & 25 & 3.8 & 38 & 25 & 3.8 & 47 \\
\hline 15 & 103 & 4.3 & 21 & 103 & 4.7 & 117 & 103 & 4.7 & 216 \\
\hline 31 & 414 & 5.4 & 28 & 414 & 5.8 & 233 & 414 & 5.9 & 849 \\
\hline 63 & 1659 & 6.6 & 34 & 1659 & 6.8 & 328 & 1659 & 6.9 & 2142 \\
\hline 127 & 6639 & 8.2 & 40 & 6639 & 7.9 & 395 & 6639 & 8.0 & 3480 \\
\hline 255 & 26560 & 9.7 & 46 & 26560 & 9.0 & 454 & 26560 & 9.0 & 4396 \\
\hline
\end{tabular}

The MGMF1 preconditioning algorithm for the above anisotropic problems can be summarized as follows: 


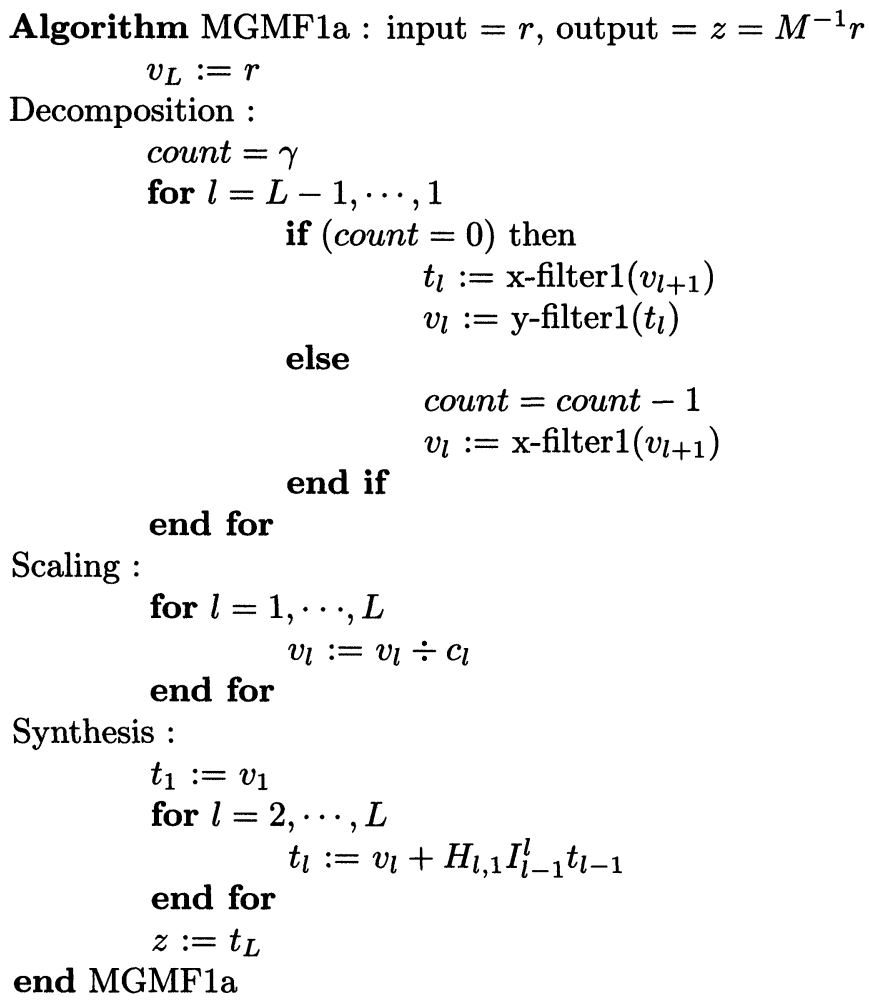

Next we show numerical results using the multigrid MF (MGMF1a) preconditioner in conjunction with the conjugate gradient method. Again, we use the standard 5 -point discretization on a uniform square mesh with $h=1 /(n+1)$ and the forcing function $f(x, y)$ is such that the solution is $u=x(x-1) y(y-1) e^{x y}$. The stopping criterion used is $\left\|r^{k}\right\| /\left\|r^{0}\right\| \leq 10^{-5}$ and the initial guess is 0 . The iteration counts for different $h$ and $\alpha$ are shown in Table 2.

TABLE 2

Iteration counts for different $\alpha$ and $n$

\begin{tabular}{|r||r|r|r||r|r|r||r|r|r|}
\hline \multicolumn{1}{|c||}{} & \multicolumn{4}{c||}{$\alpha=10$} & \multicolumn{3}{c||}{$\alpha=100$} & \multicolumn{3}{c|}{$\alpha=1000$} \\
\hline$n$ & A & MGMF1a & MGMF1 & A & MGMF1a & MGMF1 & A & MGMF1a & MGMF1 \\
\hline \hline 7 & 23 & 11 & 18 & 17 & 7 & 19 & 13 & 6 & 20 \\
\hline 15 & 48 & 13 & 26 & 41 & 10 & 41 & 27 & 9 & 44 \\
\hline 31 & 97 & 15 & 32 & 90 & 12 & 64 & 63 & 12 & 84 \\
\hline 63 & 197 & 16 & 36 & 187 & 13 & 83 & 126 & 13 & 140 \\
\hline 127 & 405 & 19 & 41 & 388 & 15 & 95 & 258 & 15 & 193 \\
\hline 255 & 839 & 20 & 45 & 812 & 17 & 106 & 608 & 17 & 224 \\
\hline
\end{tabular}

The numerical results show that this scheme works very well for a wide range of $\alpha$. A similar scheme can be applied to the case when $\alpha<1$. It should be noted that the algorithm can also be applied to more general anisotropic problems (e.g., variable coefficients) in the same way that the semicoarsening technique in MG is used (e.g., by averaging coefficients) [18]. 
4. MF preconditioners for the biharmonic equation. Consider the following biharmonic equation in two dimensions:

$$
-\triangle^{2} u=f \quad \text { in } \Omega=[0,1]^{2}
$$

with first boundary conditions:

$$
\left.u(x, y)\right|_{\Gamma}=g(x, y)
$$

and

$$
\frac{\partial u}{\partial n}=b(x, y)
$$

We discretize this equation using a 13-point second-order centered finite difference approximation with $h=1 /(n+1)$ :

$$
\begin{aligned}
20 u_{i, j} & -8\left(u_{i+1, j}+u_{i-1, j}+u_{i, j+1}+u_{i, j-1}\right) \\
& +2\left(u_{i+1, j+1}+u_{i-1, j+1}+u_{i+1, j-1}+u_{i-1, j-1}\right) \\
& +u_{i+2, j}+u_{i-2, j}+u_{i, j+2}+u_{i, j-2}=h^{4} f_{i}, j
\end{aligned}
$$

for $i, j=2, n-1$. The difference equation for $i=1$, and $j=3, \cdots, n-2$ is:

$$
\begin{aligned}
21 u_{1, j} & -8\left(u_{2, j}+u_{1, j+1}+u_{1, j-1}\right)+2\left(u_{2, j+1}+u_{2, j-1}\right)+u_{3, j}+u_{1, j+2}+u_{1, j-2} \\
& =h^{4}\left(f_{i}, j+8 g_{0, j}-2\left(g_{0, j+1}+g_{0, j-1}\right)-2 h b_{0, j}\right)
\end{aligned}
$$

since

$$
\frac{\partial u}{\partial n}=-\frac{\partial u}{\partial x} \quad \text { on } x=0
$$

Using central differencing, we get

$$
-\frac{\left(u_{1, j}-u_{-1, j}\right)}{2 h}=b_{0, j}
$$

Also, at $i=j=1$, we have

$$
\begin{aligned}
22 u_{1,1} & -8\left(u_{2,1}+u_{1,2}\right)+2\left(u_{2,2}\right)+u_{3, j}+u_{1,3} \\
& =h^{4}\left(f_{i}, j+8\left(g_{0, j}+g_{1,0}\right)-2\left(g_{0, j+1}+g_{0, j-1}+g_{2,0}\right)-2 h\left(b_{0,1}+b_{1,0}\right)\right) .
\end{aligned}
$$

The difference equations for other near boundary grid points can be derived similarly.

To derive MF preconditioners, we have to estimate the eigenvalues of the biharmonic operator. To do so, we apply Fourier analysis to the operator with modified boundary conditions, namely,

$$
\left.u(x, y)\right|_{\Gamma}=0 \quad \text { and } \quad \frac{\partial^{2} u}{\partial n^{2}}=0 .
$$

Based on analyzing this problem, we can estimate the eigenvalues of $\hat{A}$ by

$$
\hat{A}(j, k) \approx 4-2(\cos (i \pi h)+\cos (j \pi h))^{2}
$$

which is the square of that for the Poisson equation. 
TABLE 3

Condition number for SGMF preconditioning for the biharmonic equation.

\begin{tabular}{|r||r|r|r|r|}
\hline$n$ & No preconditioning & SGMF1b & SGMF2b & SGMF3b \\
\hline \hline 7 & 690 & 25 & 5.3 & 17 \\
\hline 15 & $1.1 \times 10^{4}$ & 108 & 5.6 & 66 \\
\hline 31 & $1.7 \times 10^{5}$ & 438 & 7.2 & 256 \\
\hline 63 & $2.8 \times 10^{6}$ & 1814 & 8.7 & 1017 \\
\hline 127 & $4.4 \times 10^{7}$ & 7367 & 10.2 & 4061 \\
\hline 255 & $7.0 \times 10^{8}$ & 29705 & 11.7 & 16238 \\
\hline
\end{tabular}

Since the eigenvalues in $B_{l}$ for this equation behave like $O\left(h_{l}^{-4}\right)$, a natural extension of the MF preconditioner involves changing the scaling recurrence $c_{l+1}=4 c_{l}$ to $c_{l+1}=16 c_{l}$ (again, $h_{l} \approx 2 h_{l+1}$ is assumed). In Table 3 , we show the result of the Fourier analysis on the MF-preconditioned biharmonic equation. In the table, SGMF1b, SGMF2b, and SGMF3b represent the original SGMF1, SGMF2, and SGMF3 preconditioners with the new scaling.

We see that the condition number of $A$ grows about 16 times with each halving of $h$. The use of SGMF1b has effectively helped to reduce the condition number. Nevertheless, SGMF2b helps to reduce the condition number even more dramatically.

To verify the Fourier results, we implement the SGMF1b, SGMF2b and SGMF3b preconditioners for the biharmonic equation where the $f(x, y), g(x, y)$ and $b(x, y)$ are such that the solution is $u=x(x-1) y(y-1) \sin (\pi x) \sin (\pi y)$. The stopping criterion is $\left\|r^{k}\right\| /\left\|r^{0}\right\| \leq 10^{-6}$ and the initial guess is zero. The iteration counts are shown in Table 4.

TABLE 4

Iteration counts for SGMF-preconditioned $\mathrm{PCG}$ for the biharmonic equation.

\begin{tabular}{|r||r|r|r|r|}
\hline$n$ & No preconditioning & SGMF1b & SGMF2b & SGMF3b \\
\hline \hline 7 & 10 & 9 & 10 & 9 \\
\hline 15 & 42 & 17 & 12 & 16 \\
\hline 31 & 160 & 36 & 14 & 30 \\
\hline 63 & 586 & 82 & 17 & 57 \\
\hline 127 & 2218 & 177 & 23 & 113 \\
\hline 255 & 8587 & 366 & 33 & 220 \\
\hline
\end{tabular}

Next we show (in Table 5) the iteration counts when the multigrid formulation of SGMF1b, SGMF2b, and SGMF3b (i.e., MGMF1b, MGMF2b, and MGMF3b) are applied to the same problem.

TABLE 5

Iteration counts for MGMF-preconditioned PCG for the biharmonic equation.

\begin{tabular}{|r||r|r|r|r|}
\hline$n$ & No preconditioning & MGMF1b & MGMF2b & MGMF3b \\
\hline \hline 7 & 10 & 10 & 10 & 10 \\
\hline 15 & 42 & 27 & 22 & 24 \\
\hline 31 & 160 & 40 & 29 & 32 \\
\hline 63 & 586 & 56 & 30 & 37 \\
\hline 127 & 2218 & 80 & 35 & 40 \\
\hline 255 & 8587 & 120 & 43 & 48 \\
\hline
\end{tabular}

We observe a close correlation between the numerical and Fourier results for the SGMF preconditioners. Indeed, SGMF2b improves significantly over SGMF1b with 
only a little increase in cost per iteration. SGMF3b improves somewhat over SGMF1b but is still not good enough compared to SGMF2b. Therefore, SGMF2b requires the fewest operation counts out of the three. Looking into the numerical results for the MGMF preconditioners, we first observe that both MGMF1b and MGMF3b give better convergence rates than their SGMF counterparts. We cannot explain why this is the case, nor can we explain why MGMF3b performs much better than predicted by the corresponding Fourier results. Finally, with a little arithmetic, it is not difficult to show that MGMF3b gives the fewest overall operation counts.

5. MF preconditioners for problems with locally refined grids. In this section, we shall consider the application of the MF preconditioners to second-order elliptic problems with local mesh refinement. Such mesh refinements are necessary for accurate modeling of problems with various types of singular behavior. We consider the discretization scheme for locally mesh refined grids by McCormick and Thomas [16]. This discretization scheme was motivated by the desire to preserve the highly regular grid structure (to maintain efficiency on parallel computer architectures), as well as to satisfy the need for local resolution in many physical models. For example, the mesh in Fig. 1 would be effective if the forcing function $f(x, y)$ behaves like a $\delta$ function distribution at the points $(1,1)$ and $(n, n)$ (both lower left and upper right corners).

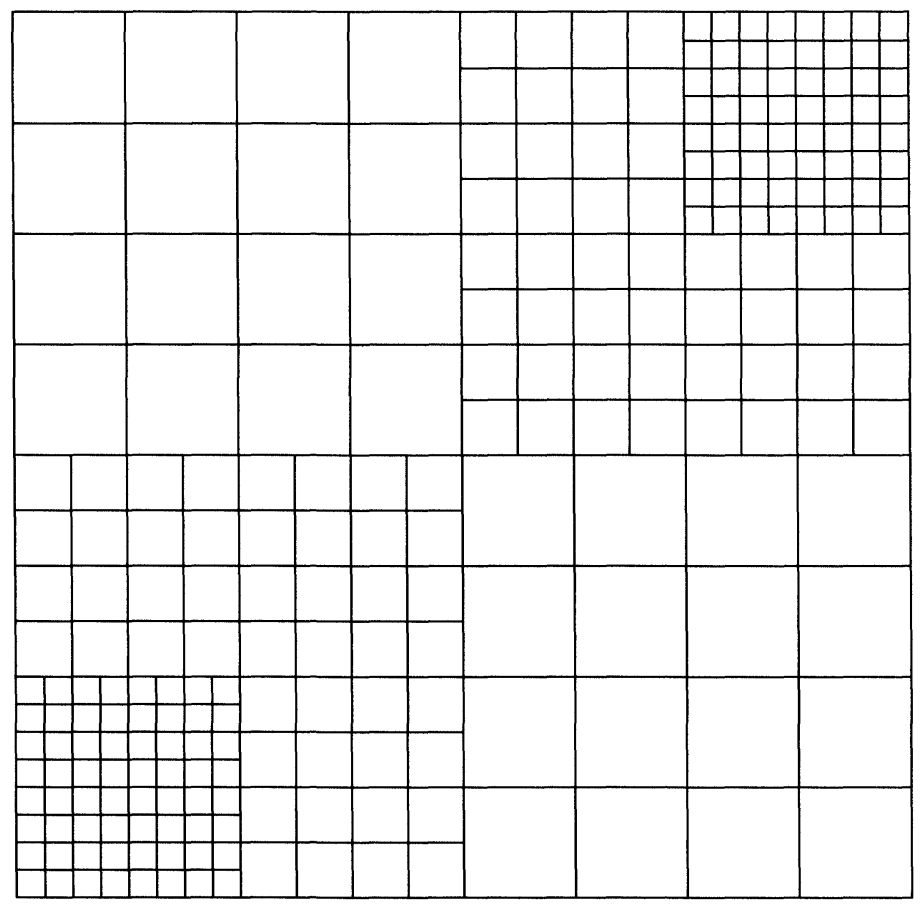

FIG. 1. Locally refined grids-an example.

The Fourier analysis cannot be applied here because of the presence of nonuniform grids. However, as was shown in our previous paper [13], the parallel multilevel preconditioner proposed by Bramble, Pasciak, and Xu [6] can be considered as a special case of MF preconditioners with appropriately chosen filters. We can borrow the finite element analysis result from them and we would expect the MGMF precon- 
ditioners to be effective also for meshes with local refinement. Below we show the MGMF algorithm for this problem. Here $\hat{I}_{i}^{j}$ and $\hat{H}_{l}$ are restriction (or interpolation) and elementary filtering operators restricted to the locally refined grids only. Moreover, we can use the same recurrence relation $c_{l}=4 c_{l+1}$ and we have the following algorithm:

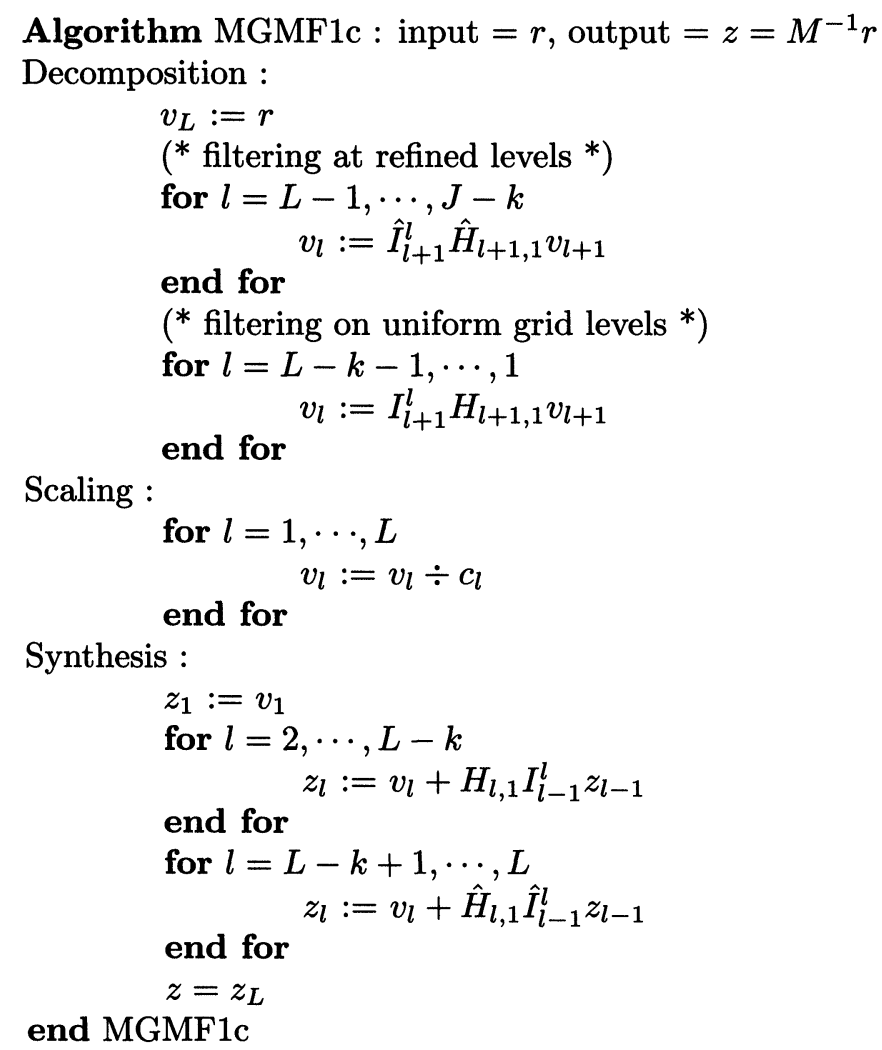

We solve a Poisson equation on the grid

- shown in Fig. 1 but with refinement only at the upper right corner and the forcing function is $f(x, y)=2^{-l} \delta(1-h, 1-h)$, and

- shown in Fig. 1 and the forcing function $f(x, y)=2^{-l}(\delta(h, h)+\delta(1-h, 1-h))$, where $l$ is the number of level of refinements used and $h$ is the grid size for the nonrefined grid.

We use the discretization scheme for the domain and the interfaces proposed by McCormick and Thomas [16] for aligned grids. The stopping criterion and initial guess are the same as before. The iteration counts for different number of levels and different $h$ are given in Tables 6 and 7. The iteration counts for the unpreconditioned conjugate-gradient (CG) method and the parallel multilevel preconditioner (BPX) [6] are also included for comparison purposes.

The tables show the effectiveness of the MF preconditioner compared to the unpreconditioned CG method and the PCG method with parallel multilevel preconditioner (BPX). The convergence rates seem to be quite insensitive to the number of refinement levels used. 
TABLE 6

Iteration counts for the Poisson equation with refinements at upper right corner only.

\begin{tabular}{|r||r|r|r|r|}
\hline$n$ & No. of levels & CG & MGMF1c & BPX \\
\hline \hline 15 & 0 & 26 & 9 & 12 \\
\hline 15 & 1 & 37 & 10 & 14 \\
\hline 15 & 2 & 45 & 11 & 16 \\
\hline 15 & 3 & 53 & 12 & 17 \\
\hline \hline 31 & 0 & 48 & 9 & 13 \\
\hline 31 & 1 & 70 & 10 & 15 \\
\hline 31 & 2 & 88 & 11 & 17 \\
\hline 31 & 3 & 109 & 12 & 18 \\
\hline \hline 63 & 0 & 84 & 10 & 14 \\
\hline 63 & 1 & 126 & 11 & 15 \\
\hline 63 & 2 & 166 & 11 & 17 \\
\hline 63 & 3 & 210 & 12 & 19 \\
\hline \hline 127 & 0 & 133 & 10 & 14 \\
\hline 127 & 1 & 219 & 11 & 15 \\
\hline 127 & 2 & 309 & 12 & 17 \\
\hline 127 & 3 & 395 & 13 & 19 \\
\hline
\end{tabular}

TABLE 7

Iteration counts for the Poisson equation with refinements at both corners.

\begin{tabular}{|r||r|r|r|r|}
\hline$n$ & No. of levels & CG & MGMF1c & BPX \\
\hline \hline 15 & 0 & 26 & 9 & 12 \\
\hline 15 & 1 & 54 & 11 & 15 \\
\hline 15 & 2 & 63 & 12 & 17 \\
\hline 15 & 3 & 75 & 16 & 18 \\
\hline \hline 31 & 0 & 48 & 9 & 13 \\
\hline 31 & 1 & 86 & 11 & 16 \\
\hline 31 & 2 & 117 & 13 & 17 \\
\hline 31 & 3 & 140 & 13 & 19 \\
\hline \hline 63 & 0 & 84 & 10 & 14 \\
\hline 63 & 1 & 126 & 12 & 16 \\
\hline 63 & 2 & 190 & 12 & 18 \\
\hline 63 & 3 & 235 & 14 & 19 \\
\hline \hline 127 & 0 & 133 & 10 & 14 \\
\hline 127 & 1 & 204 & 12 & 16 \\
\hline 127 & 2 & 297 & 13 & 18 \\
\hline 127 & 3 & 391 & 14 & 20 \\
\hline
\end{tabular}

6. MF preconditioners for Schur complement systems. Consider solving a two-dimensional second-order elliptic problem on a domain divided into two subdomains by an interface. If we use a 5-point discretization and order unknowns in the subdomains $\Omega_{1}$ and $\Omega_{2}$ first followed by those on the interface $\Gamma_{3}$, we obtain the following linear system:

$$
A u=\left[\begin{array}{ccc}
A_{1} & 0 & A_{13} \\
0 & A_{2} & A_{23} \\
A_{31} & A_{32} & A_{3}
\end{array}\right]\left[\begin{array}{l}
u_{1} \\
u_{2} \\
u_{3}
\end{array}\right]=\left[\begin{array}{l}
f_{1} \\
f_{2} \\
f_{3}
\end{array}\right]
$$

By applying block Gaussian elimination to eliminate the unknowns $u_{1}$ and $u_{2}$, we obtain the following system for the interface unknowns $u_{3}$ :

$$
S u_{3}=\tilde{f}_{3}
$$


where

$$
S=A_{3}-A_{31} A_{1}^{-1} A_{13}-A_{32} A_{2}^{-1} A_{23}
$$

and

$$
\tilde{f}_{3}=f_{3}-A_{31} A_{1}^{-1} f_{1}-A_{32} A_{2}^{-1} f_{2} .
$$

A standard approach in domain decomposition methods is to solve the Schur complement system $S u_{3}=\tilde{f}_{3}$ with the preconditioned conjugate gradient method. Many preconditioners have been proposed in the literature [7]. A typical one is Dryja's preconditioner [9], which is defined to be the square root of the negative one-dimensional Laplacian and which can be inverted by the use of FFTs in $O(n \log n)$ time, where $n$ is the number of unknowns on the interface. Recently, Smith and Widlund [19] proposed a hierarchical basis preconditioner for $S$ which is cheaper than Dryja's preconditioner, requiring only $O(n)$ work per iteration. Here we propose to use the MF preconditioner for $S$. To do this, we can retain the multilevel filtering framework and we only need to modify the scaling constants $c_{l}$ 's. We know that the eigenvalues for the Schur complement in the frequency band $B_{l}$ behaves like $O\left(h_{l}^{-1}\right)$ [9]. Therefore, it is sufficient to use the recurrence $c_{l+1}=2 c_{l}$. In Table 8 we compare the number of iterations to obtain convergence for different $n$ for the Poisson equation on a rectangular $2 n \times n$ grid decomposed into two equal subdomains.

TABLE 8

Iteration count versus $n$.

\begin{tabular}{|c||c|c|c|c|c|}
\hline$n$ & No precond. & Dryja & MGMF1 & MGMF2 & HB \\
\hline \hline 7 & 4 & 4 & 4 & 4 & 4 \\
\hline 15 & 8 & 6 & 7 & 6 & 7 \\
\hline 31 & 16 & 6 & 9 & 7 & 8 \\
\hline 63 & 27 & 6 & 9 & 7 & 10 \\
\hline 127 & 39 & 6 & 9 & 7 & 12 \\
\hline
\end{tabular}

We observe that MGMF2 performs better than MGMF1 and the hierarchical basis (HB) preconditioner. All but the HB preconditioner seem to show convergence rates independent of $n$. Although we cannot prove spectral equivalence for the MGMF preconditioners, an $O(\log n)$ upper bound for the condition number for the MGMF1 preconditioned system can be proved and details of such a proof can be found in [24]. We also observe that the MGMF2 preconditioner performs almost as well as Dryja's preconditioner. The MGMF appears to offer convergence rates comparable to Dryja's preconditioner and at the same time is relatively easy to use and costs about the same as the $\mathrm{HB}$ preconditioner.

7. Conclusion. In our previous paper [13] and the first part of the present paper we show the competitiveness of the MF preconditioners compared with some other preconditioners such as the hierarchical basis preconditioner, multigrid preconditioner and others. In this paper we have further demonstrated the ease with which we can extend the MF preconditioners to effectively solve other more general elliptic problems. The flexibility of filter and scaling block design offers different ways of achieving a high degree of efficiency for these problems. 


\section{REFERENCES}

[1] O. AxelsSon, An algebraic framework for multilevel methods, Report 8820, Department of Mathematics, Catholic University, Toernooiveld, 6525 ED Nijmegen, the Netherlands, 1988.

[2] O. Axelsson AND P. VAssilevski, Algebraic multilevel preconditioning methods, I, Report 8811, Department of Mathematics, Catholic University, Toernooiveld, 6525 ED Nijmegen, the Netherlands, 1988.

[3] — Algebraic multilevel preconditioning methods, II, Report 1988-15, Institute for Scientific Computation, University of Wyoming, Laramie, WY, 1988.

[4] R. E. BANK, T. F. DUPONT, AND H. YSERENTANT, The hierarchical basis multigrid method, Numer. Math., 52 (1988), pp. 427-458.

[5] G. BIRKhoff AND R. E. LyNCH, Numerical Solutions for Elliptic Problems, Society for Industrial and Applied Mathematics, Philadelphia, 1984.

[6] J. H. Bramble, J. E. Pasciak, And J. Xu, Parallel multilevel preconditioners, Math. Comp., 55 (1990), pp. 1-22.

[7] T. F. Chan, R. Glowinski, J. Periaux, O. B. Widlund, eds., Domain Decomposition Methods for Partial Differential Equations, Society for Industrial and Applied Mathematics, Philadelphia, 1989.

[8] T. F. Chan, Jay C. C. Kuo, And C. H. Tong, Parallel elliptic preconditioners: Fourier analysis and performance on the Connection Machine, Comput. Phys. Comm., 53 (1989), pp. 237-252.

[9] M. DRYJA, A capacitance matrix method for Dirichlet problem on polygon region, Numer. Math., 39 (1982), pp. 51-54.

[10] A. Greenbaum, C. Li, and H. Z. Chao, Parallelizing preconditioned conjugate gradient algorithms, Comput. Phys. Comm., 53 (1989), pp. 295-309.

[11] W. HACKBUSCH, The frequency decomposition multi-grid method, Numer. Math., 56 (1989), pp. 229-245.

[12] V. E. Henson AND W. L. BRIGGs, Wavelets: What are they and what do they have to do with Multigrid?, Copper Mountain Conference on Iterative Methods, April 1-5, Copper Mountain, CO, 1990.

[13] C.-C. J. Kuo, T. F. ChAn, And C. H. Tong, Multilevel filtering elliptic preconditioners, SIAM J. Matrix Anal. Appl., 11 (1990), pp. 403-429.

[14] Y. A. KuZnetsov, Multigrid domain decomposition methods for elliptic problems, Proceedings VIII International Conference on Computational Methods for Applied Science and Eng. Vol. 2, Versailles, France, 1987, pp. 605-616.

[15] R. E. LYNCH AND J. R. RICE, A high-order difference method for differential equations, Math. Comp., 34 (1980), pp. 333-372.

[16] S. MCCoRmick AND J. Thomas, The fast adaptive composite grid (FAC) method for elliptic equations, Math. Comp., 46 (1986), pp. 439-456.

[17] S. MCCormick, Multilevel Adaptive Methods for Partial Differential Equations, Society for Industrial and Applied Mathematics, Philadelphia, 1989.

[18] W. A. Mulder, Multigrid Alignment and Euler's Equation, in Proc. Fourth Copper Mountain Conference on Multigrid Methods, Society for Industrial and Applied Mathematics, Philadelphia, 1989, pp. 348-364.

[19] B. Smith AND O. WidLund, A domain decomposition algorithm using a hierarchical basis, SIAM J. Sci. Statist. Comput., 11 (1990), pp. 1212-1220.

[20] G. STrANG, Wavelets and dilation equations : A brief introduction, SIAM Rev., 31 (1989), pp. 614-627.

[21] G. Strang And G. J. Fix, An Analysis of the Finite Element Method, Prentice-Hall Ser. Automat. Comput., 1973.

[22] Charles H. Tong, The preconditioned conjugate gradient method on the connection machine, Internat. J. High Speed Comput., 2nd Issue: Scientific Applications of the Connection Machine, 1989.

[23] C. TonG, T. F. ChAN, AND C. C. J. KUO, Multilevel filtering preconditioners-extension to more general elliptic problems, UCLA CAM Report 90-12, University of California, Los Angeles, CA, 1990

[24] - A domain decomposition preconditioner based on a change to the multilevel nodal basis, UCLA CAM Report 90-20, University of California, Los Angeles, CA, 1990.

[25] P. VASSILEVSKI, Iterative methods for solving finite element equations based on multilevel splitting of the matrix, Bulgarian Academy of Science, Sofia, Bulgaria, 1987, preprint. 
[26] P. Wesseling, A survey of Fourier smoothing analysis results, Third European Conference on Multigrid Methods, Oct. 1-3, 1990.

[27] D. M. Young AND B. R. VonA, Parallel multilevel methods, CNA-243, University of Texas, Austin, TX, May 1990.

[28] H. YSERENTANT, On the multi-level splitting of finite element spaces, Numer. Math., 49 (1986), pp. 379-412. 